\title{
Finite-Time Stability of Uncertain Nonlinear Systems with Time-Varying Delay
}

\author{
Jingting Hu, ${ }^{1}$ Guixia Sui, ${ }^{2}$ Shengli $\mathrm{Du}^{3}$ and Xiaodi $\mathrm{Li}^{4}$ \\ ${ }^{1}$ School of Mathematical Sciences, University of Jinan, Jinan 250022, China \\ ${ }^{2}$ Primary Education Department, Jinan Preschool Education College, Jinan 250307, China \\ ${ }^{3}$ College of Automation, Beijing University of Technology, Beijing 100124, China \\ ${ }^{4}$ School of Mathematics and Statistics, Shandong Normal University, Jinan 250014, China \\ Correspondence should be addressed to Jingting Hu; jingthu74@163.com and Xiaodi Li; sodymath@163.com
}

Received 7 July 2017; Accepted 13 September 2017; Published 15 October 2017

Academic Editor: J.-C. Cortés

Copyright (c) 2017 Jingting Hu et al. This is an open access article distributed under the Creative Commons Attribution License, which permits unrestricted use, distribution, and reproduction in any medium, provided the original work is properly cited.

\begin{abstract}
The problem of finite-time stability for a class of uncertain nonlinear systems with time-varying delay and external disturbances is investigated. By using the Lyapunov stability theory, sufficient conditions for the existence of finite-time state feedback controller for this class of systems are derived. The results can be applied to finite-time stability problems of linear time-delay systems with parameter uncertainties and external disturbances. Finally, two numerical examples are given to demonstrate the effectiveness of the obtained theoretical results.
\end{abstract}

\section{Introduction}

Time delays are frequently encountered in various engineering and physical systems such as chemical systems, networked control systems, and power systems. It is well known that time delays will deteriorate the system's performance and even make the system unstable. Therefore, it is important to study the stability issue of time-delay systems. In recent years, the stability problem of linear systems has been well addressed $[1,2]$. Some results of nonlinear systems with time-varying delay can be found in [3-9] and the references therein. On the other hand, parameter uncertainties cannot be avoided in a control system due to modeling errors, external disturbances, and linearization approximations, which are the main causes of instability and poor performance of control systems. This is also the reason why some authors study stochastic differential equations $[10,11]$. Many authors studied the models with parameter uncertainties $[12,13]$. The stability of uncertain systems has also gained much research attention $[14,15]$.

It should be noted that most of the stability results in existing literature are the classical Lyapunov asymptotic stability, which is defined over an infinite time interval [16-18]. However, in practice, it is more meaningful to investigate the finite-time stability problem. Different from Lyapunov stability, finite-time stability means that the system is Lyapunov stable and its trajectories tend to zero in a finite time. Finite-time stability leads to better robustness and disturbance rejection properties [19, 20]. In [19], the authors presented a necessary and sufficient condition for finite-time stability of continuous-time autonomous system. After that, much attention has been given to finite-time stability and stabilization. For the delay-free case, many results on finitetime stability can be found in [21-28]. The authors in [24] investigated finite-time stabilization of a class of nonlinear systems with parametric uncertainties and proposed a controller by using Lyapunov, backstepping, and input-to-state stability techniques. Reference [25] studied finite-time stability for continuous-time systems with uniqueness of solutions in forward time and obtained a necessary and sufficient condition for finite-time stability for this class of systems. Recently, huge efforts have been paid to the finite-time stability or stabilization problems for time-delay systems [2934]. In [30], the authors provided a theoretical result on finitetime stability by using Lyapunov functional and showed how to obtain finite-time stabilization of linear systems with time delays. The authors in [32] investigated the finite-time stability and designed a finite-time $H_{\infty}$ controller for a class 
of nonlinear time-delay Hamiltonian systems. Meanwhile, the finite-time stability or synchronization problem of neural networks was studied in [35-42]. It is worth noting that most of the results in literature focus on the finite-time stability for systems with or without time delays. Until now, there are rare results on the finite-time stability for nonlinear systems in the presence of time delays, parameter uncertainties, and external disturbances [43]. Note that the systems studied in $[24,25]$ are without time delays, and those in [30,32] are with time delays but without parameter uncertainties and external disturbances.

In this paper, motivated by the above discussions, we shall address the finite-time stability problem of nonlinear systems with time-varying delay, parameter uncertainties, and external disturbances. By using a designed delayed feedback controller, some sufficient conditions for finitetime stability for such a class of systems are established. The main results of this paper are as follows. First, finite-time stability problem for uncertain nonlinear systems with time delays and disturbances is formulated. Second, a delayed feedback controller is designed to achieve the finite-time stability for a class of uncertain nonlinear systems with time delays and external disturbances based on the finite-time Lyapunov stability theory. Sufficient conditions are derived for the finite-time stability of the studied system. Finally, two numerical examples are provided to show the effectiveness of the proposed results.

The remainder of the paper is organized as follows. In Section 2, the model of uncertain nonlinear systems with time-varying delay and external disturbances is presented. Some necessary preliminaries are also given in this section. The finite-time stability criteria are obtained in Section 3. Then, two numerical simulation examples are provided to show the effectiveness of our results in Section 4. Finally, some conclusions are drawn in Section 5.

\section{Preliminaries}

Notations 1 . Let $\mathbb{R}$ be the set of real numbers and let $\mathbb{R}^{n}$ and $\mathbb{R}^{n \times m}$ be the $n$-dimensional and $n \times m$-dimensional real spaces equipped with the Euclidean norm $\|\cdot\|$, respectively. $\mathbb{Z}_{+}$is the set of positive integer numbers. $\lambda_{\max }(P)$ represents the maximum eigenvalue of symmetric matrix $P . P>0(P<0)$ denotes that matrix $P$ is a symmetric and positive definite (negative definite) matrix. Let $I$ denote the identity matrix with appropriate dimensions. For any interval $J \subseteq R$, set $S \subseteq R^{k}(1 \leq k \leq n), C(J, S)=\{\varphi: J \rightarrow S$ is continuous $\}$.

Consider the following uncertain nonlinear system with time-varying delay and external disturbances:

$$
\begin{aligned}
\dot{x}(t)= & (A+\Delta A) x(t)+(B+\Delta B) f(x(t-\tau(t))) \\
& +E \omega(t),
\end{aligned}
$$

where $x(t)=\left(x_{1}(t), x_{2}(t), \ldots, x_{n}(t)\right)^{T} \in \mathbb{R}^{n}$ is the state vector. $E \in \mathbb{R}^{n \times m}, A, B \in \mathbb{R}^{n \times n}$ are constant matrices. $\Delta A$ and $\Delta B \in \mathbb{R}^{n \times n}$ are unknown matrices standing for parameter uncertainties satisfying $\Delta A^{T} \Delta A \leq \alpha I, \Delta B^{T} \Delta B \leq \beta I$, and $\alpha, \beta>0 . \omega(t) \in \mathbb{R}^{m}$ is the external disturbances vector satisfying $\omega^{T}(t) \omega(t) \leq d$, and $d>0 . f(x(t))=\left(f_{1}\left(x_{1}(t)\right)\right.$, $\left.f_{2}\left(x_{2}(t)\right), \ldots, f_{n}\left(x_{n}(t)\right)\right)^{T} \in \mathbb{R}^{n} ; \tau(t)$ is a time-varying delay, which satisfies $\dot{\tau}(t) \leq \mu<1 . f: \mathbb{R}^{n} \rightarrow \mathbb{R}^{n}$ is a nonlinear function satisfying the Lipschitz condition; namely, there exists a positive constant $L$, for all $x, y \in \mathbb{R}^{n}$, such that

$$
\|f(y)-f(x)\|^{2} \leq L\|y-x\|^{2} .
$$

The initial condition of system (1) is assumed to be

$$
x(t)=\phi(t), \quad \forall t \in[-\tau, 0],
$$

where $\phi(t) \in C\left([-\tau, 0], R^{n}\right)$.

System (1) with a control input is given as

$$
\begin{aligned}
\dot{x}(t)= & (A+\Delta A) x(t)+(B+\Delta B) f(x(t-\tau(t))) \\
& +E \omega(t)+u(t),
\end{aligned}
$$

where $u(t)$ denotes the control input and is given by

$$
u(t)=u_{1}(t)+u_{2}(t)
$$

with

$$
\begin{aligned}
u_{1}(t)= & -k_{1} x(t)-\lambda \operatorname{sign}(x(t))|x(t)|^{\eta} \\
& -\lambda\left(k_{2} \int_{t-\tau(t)}^{t} x^{T}(s) x(s) d s\right)^{(1+\eta) / 2} \frac{x(t)}{\|x(t)\|^{2}}, \\
u_{2}(t)= & -\frac{s_{4}}{2} E E^{T} x(t)-\frac{s_{4}^{-1} d}{2\|x(t)\|^{2}} x(t),
\end{aligned}
$$

and $|x(t)|^{\eta}=\left(\left|x_{1}(t)\right|^{\eta},\left|x_{2}(t)\right|^{\eta}, \ldots,\left|x_{n}(t)\right|^{\eta}\right)^{T}, \lambda>0,0<\eta<$ $1, s_{4}>0, k_{1}$ and $k_{2}$ are constants to be determined later, and $\operatorname{sign}(x(t))=\operatorname{diag}\left(\operatorname{sign}\left(x_{1}(t)\right), \operatorname{sign}\left(x_{2}(t)\right), \ldots, \operatorname{sign}\left(x_{n}(t)\right)\right)$.

For further analysis, we first give the following definition and useful lemmas.

Definition 2 (see [30]). The equilibrium $x=0$ of system (4) is said to be finite-time stable if it is Lyapunov stable and finitetime convergent. Finite-time convergence means that, for any initial state $\varphi(s)$, there is a settling time $0<T(\varphi)<+\infty$, such that every solution $x(t, \varphi)$ of system (4) satisfies

$$
\begin{aligned}
\lim _{t \rightarrow T} x(t, \varphi) & =0, \\
x(t, \varphi) & =0, \\
& \forall t>T(\varphi) .
\end{aligned}
$$

Lemma 3 (see [19]). Assume that $V(t)$ is a continuous positive definite function satisfying the following inequality:

$$
\dot{V}(t) \leq-c V^{\alpha}(t), \quad \forall t \geq t_{0}, \quad v\left(t_{0}\right) \geq 0,
$$

where $c>0,0<\alpha<1$ are all constants. Then, for any given $t_{0} \geq 0, V(t)$ satisfies the following inequality:

$$
V^{1-\alpha}(t) \leq V^{1-\alpha}\left(t_{0}\right)-c(1-\alpha)\left(t-t_{0}\right), \quad t_{0} \leq t \leq T,
$$

and $V(t) \equiv 0, \forall t \geq T$, with $T$ given by

$$
T=t_{0}+\frac{V^{1-\alpha}\left(t_{0}\right)}{c(1-\alpha)} .
$$


Lemma 4 (see [44]). For any real matrices $A, B$, and $\Sigma$ with appropriate dimensions and a scalar $s>0$, if the matrix $\Sigma$ satisfies $0<\Sigma=\Sigma^{T}$, then the following inequality holds:

$$
A^{T} B+B^{T} A \leq s A^{T} \Sigma A+s^{-1} B^{T} \Sigma^{-1} B .
$$

Lemma 5 (see [45]). For any $a_{i} \in \mathbb{R}, i \in \mathbb{Z}_{+}$, and any real numbers $0<p \leq 1,0<q<2$, we have the following assertions:

(1) $\left|a_{1}\right|^{q}+\left|a_{2}\right|^{q}+\cdots+\left|a_{n}\right|^{q} \geq\left(\left|a_{1}\right|^{2}+\left|a_{2}\right|^{2}+\cdots+\left|a_{n}\right|^{2}\right)^{q / 2}$.

(2) $\left(\left|a_{1}\right|+\left|a_{2}\right|+\cdots+\left|a_{n}\right|\right)^{p} \leq\left|a_{1}\right|^{p}+\left|a_{2}\right|^{p}+\cdots+\left|a_{n}\right|^{p}$.

\section{Main Results}

In this section, we shall present some sufficient conditions for the finite-time stability of system (1) with control input $u(t)$ defined in (5).

Theorem 6. Assume that there exist constants $s_{1}, s_{2}, s_{3}>0$ and $k_{1}, k_{2}>0$ such that

(i) $A^{T}+A-2 k_{1} I+k_{2} I+s_{2} B B^{T}+s_{3}^{-1} I+\left(s_{1} \alpha+s_{1}^{-1}\right) I \leq 0$;

(ii) $\left(s_{2}^{-1}+s_{3} \beta\right) L-k_{2}(1-\mu) \leq 0$;

then system (1) is finite-time stable with control input $u(t)$ in (5), and the finite time is estimated by

$$
T=t_{0}+\frac{V^{(1-\eta) / 2}\left(t_{0}\right)}{\lambda(1-\eta)} .
$$

Proof. Choose the following Lyapunov functional:

$$
V(t)=x^{T}(t) x(t)+\int_{t-\tau(t)}^{t} k_{2} x^{T}(s) x(s) d s
$$

Taking the derivative of $V(t)$ along the trajectory of system (4), we have

$$
\begin{aligned}
\dot{V} & (t)=\dot{x}^{T}(t) x(t)+x^{T}(t) \dot{x}(t)+k_{2} x^{T}(t) x(t) \\
& -k_{2} x^{T}(t-\tau(t)) x(t-\tau(t))(1-\dot{\tau}(t)) \leq \dot{x}^{T}(t) \\
& \cdot x(t)+x^{T}(t) \dot{x}(t)+k_{2} x^{T}(t) x(t)-k_{2} x^{T}(t \\
& -\tau(t)) x(t-\tau(t))(1-\mu)=[(A+\Delta A) x(t) \\
& +(B+\Delta B) f(x(t-\tau(t)))+E \omega(t)+u(t)]^{T} x(t) \\
& +x^{T}(t)[(A+\Delta A) x(t)
\end{aligned}
$$

$+(B+\Delta B) f(x(t-\tau(t)))+E \omega(t)+u(t)]$

$+k_{2} x^{T}(t) x(t)-k_{2} x^{T}(t-\tau(t)) x(t-\tau(t))(1$

$-\mu)=[(A+\Delta A) x(t)+(B+\Delta B) f(x(t-\tau(t)))$

$\left.+E \omega(t)+u_{1}(t)+u_{2}(t)\right]^{T} x(t)+x^{T}(t)$

$\cdot[(A+\Delta A) x(t)+(B+\Delta B) f(x(t-\tau(t)))$

$\left.+E \omega(t)+u_{1}(t)+u_{2}(t)\right]+k_{2} x^{T}(t) x(t)$

$-k_{2} x^{T}(t-\tau(t)) x(t-\tau(t))(1-\mu)$

$=[(A+\Delta A) x(t)+(B+\Delta B) f(x(t-\tau(t)))$

$-k_{1} x(t)-\lambda \operatorname{sign}(x(t))|x(t)|^{\eta}$

$\left.-\lambda\left(k_{2} \int_{t-\tau(t)}^{t} x^{T}(s) x(s) d s\right)^{(1+\eta) / 2} \frac{x(t)}{\|x(t)\|^{2}}\right]^{T}$

$\cdot x(t)+x^{T}(t)[(A+\Delta A) x(t)$

$+(B+\Delta B) f(x(t-\tau(t)))-k_{1} x(t)$

$-\lambda \operatorname{sign}(x(t))|x(t)|^{\eta}$

$\left.-\lambda\left(k_{2} \int_{t-\tau(t)}^{t} x^{T}(s) x(s) d s\right)^{(1+\eta) / 2} \frac{x(t)}{\|x(t)\|^{2}}\right]$

$+k_{2} x^{T}(t) x(t)-k_{2} x^{T}(t-\tau(t)) x(t-\tau(t))(1$

$-\mu)+\omega^{T}(t) E^{T} x(t)+u_{2}^{T}(t) x(t)+x^{T}(t) E \omega(t)$

$+x^{T}(t) u_{2}(t)=\left[x^{T}(t)(A+\Delta A)^{T}\right.$

$+f^{T}(x(t-\tau(t)))(B+\Delta B)^{T}-k_{1} x^{T}(t)$

$-\lambda\left(|x(t)|^{\eta}\right)^{T} \operatorname{sign}^{T}(x(t))$

$\left.-\lambda\left(k_{2} \int_{t-\tau(t)}^{t} x^{T}(s) x(s) d s\right)^{(1+\eta) / 2} \frac{x^{T}(t)}{\|x(t)\|^{2}}\right] x(t)$

$+x^{T}(t)[(A+\Delta A) x(t)$

$+(B+\Delta B) f(x(t-\tau(t)))-k_{1} x(t)$

$-\lambda \operatorname{sign}(x(t))|x(t)|^{\eta}$

$\left.-\lambda\left(k_{2} \int_{t-\tau(t)}^{t} x^{T}(s) x(s) d s\right)^{(1+\eta) / 2} \frac{x(t)}{\|x(t)\|^{2}}\right]$

$+k_{2} x^{T}(t) x(t)-k_{2} x^{T}(t-\tau(t)) x(t-\tau(t))(1$

$-\mu)+2 x^{T}(t) E \omega(t)+2 x^{T}(t) u_{2}(t)=x^{T}(t)$ 


$$
\begin{aligned}
& \cdot\left[(A+\Delta A)^{T}+(A+\Delta A)-2 k_{1} I+k_{2} I\right] x(t) \\
& +\left[f^{T}(x(t-\tau(t)))(B+\Delta B)^{T} x(t)\right. \\
& \left.+x^{T}(t)(B+\Delta B) f(x(t-\tau(t)))\right]-k_{2} x^{T}(t \\
& -\tau(t)) x(t-\tau(t))(1-\mu) \\
& +\left[-\lambda\left(|x(t)|^{\eta}\right)^{T} \operatorname{sign}^{T}(x(t)) x(t)\right. \\
& \left.-\lambda x^{T}(t) \operatorname{sign}(x(t))|x(t)|^{\eta}\right] \\
& -2 \lambda\left(k_{2} \int_{t-\tau(t)}^{t} x^{T}(s) x(s) d s\right)^{(1+\eta) / 2}+2 x^{T}(t) \\
& \cdot E \omega(t)+2 x^{T}(t)\left[-\frac{s_{4}}{2} E E^{T} x(t)-\frac{s_{4}^{-1} d}{2\|x(t)\|^{2}} x(t)\right] .
\end{aligned}
$$

Based on Lemma 4, one can obtain that the following inequality holds:

$$
\begin{aligned}
x^{T} & (t) \Delta A^{T} x(t)+x^{T}(t) \Delta A x(t) \\
& \leq s_{1} x^{T}(t) \Delta A^{T} \Delta A x(t)+s_{1}^{-1} x^{T}(t) x(t) \\
& \leq s_{1} x^{T}(t) \alpha x(t)+s_{1}^{-1} x^{T}(t) x(t) \\
& =\left(s_{1} \alpha+s^{-1}\right) x^{T}(t) x(t) .
\end{aligned}
$$

Similarly, one has

$$
\begin{aligned}
f^{T} & (x(t-\tau(t)))(B+\Delta B)^{T} x(t)+x^{T}(t)(B+\Delta B) \\
& +f(x(t-\tau(t)))=f^{T}(x(t-\tau(t))) B^{T} x(t) \\
& +f^{T}(x(t-\tau(t))) \Delta B^{T} x(t)+x^{T}(t) \\
& +B f(x(t-\tau(t)))+x^{T}(t) \Delta B f(x(t-\tau(t))) \\
& =\left[f^{T}(x(t-\tau(t))) B^{T} x(t)\right. \\
& \left.+x^{T}(t) B f(x(t-\tau(t)))\right] \\
& +\left[f^{T}(x(t-\tau(t))) \Delta B^{T} x(t)\right. \\
& \left.+x^{T}(t) \Delta B f(x(t-\tau(t)))\right] \leq\left[s_{2} x^{T}(t) B B^{T} x(t)\right. \\
& \left.+s_{2}^{-1} f^{T}(x(t-\tau(t))) f(x(t-\tau(t)))\right] \\
+ & {\left[s_{3} f^{T}(x(t-\tau(t))) \Delta B^{T} \Delta B f(x(t-\tau(t)))\right.} \\
& \left.+s_{3}^{-1} x^{T}(t) x(t)\right] \leq\left[s_{2} x^{T}(t) B B^{T} x(t)\right. \\
& \left.+s_{2}^{-1} L x^{T}(t-\tau(t)) x(t-\tau(t))\right] \\
+ & {\left[s_{3} \beta L x^{T}(t-\tau(t)) x(t-\tau(t))+s_{3}^{-1} x^{T}(t) x(t)\right] }
\end{aligned}
$$

$$
\begin{aligned}
& \quad=x^{T}(t)\left(s_{2} B B^{T}+s_{3}^{-1} I\right) x(t)+x^{T}(t-\tau(t))\left(s_{2}^{-1} L I\right. \\
& \left.\quad+s_{3} \beta L I\right) x(t-\tau(t)), \\
& 2 x^{T}(t) E \omega(t) \leq s_{4} x^{T}(t) E E^{T} x(t)+s_{4}^{-1} \omega^{T}(t) \omega(t) .
\end{aligned}
$$

Also, we can derive the following inequality:

$$
\begin{aligned}
- & \lambda\left(|x(t)|^{\eta}\right)^{T} \operatorname{sign}^{T}(x(t)) x(t)-\lambda x^{T}(t) \operatorname{sign}(x(t)) \\
& \cdot|x(t)|^{\eta}=-\lambda\left(|x(t)|^{\eta}\right)^{T}|x(t)|-\lambda|x(t)|^{T}|x(t)|^{\eta} \\
& =-2 \lambda|x(t)|^{T}|x(t)|^{\eta}=-2 \lambda\left(\left|x_{1}(t)\right|^{1+\eta}\right. \\
& \left.+\left|x_{2}(t)\right|^{1+\eta}+\cdots+\left|x_{n}(t)\right|^{1+\eta}\right) \\
& =-2 \lambda\left(\left|x_{1}(t)\right|^{2((1+\eta) / 2)}+\left|x_{2}(t)\right|^{2((1+\eta) / 2)}+\cdots\right. \\
& \left.+\left|x_{n}(t)\right|^{2((1+\eta) / 2)}\right) \leq-2 \lambda\left(\left|x_{1}(t)\right|^{2}+\left|x_{2}(t)\right|^{2}+\cdots\right. \\
& \left.+\left|x_{n}(t)\right|^{2}\right)^{(1+\eta) / 2}=-2 \lambda\left|x^{T}(t) x(t)\right|^{(1+\eta) / 2} .
\end{aligned}
$$

Substituting (15)-(17) into (14), it yields that

$$
\begin{aligned}
& \dot{V}(t) \leq x^{T}(t)\left[A^{T}+A-2 k_{1} I+k_{2} I+\left(s_{1} \alpha+s_{1}^{-1}\right) I\right] \\
& \cdot x(t)+x^{T}(t)\left(s_{2} B B^{T}+s_{3}^{-1} I\right) x(t)+x^{T}(t-\tau(t)) \\
& \cdot\left(s_{2}^{-1} L I+s_{3} \beta L I\right) x(t-\tau(t))-k_{2}(1-\mu) x^{T}(t \\
& -\tau(t)) x(t-\tau(t))-2 \lambda\left|x(t)^{T} x(t)\right|^{(1+\eta) / 2} \\
& -2 \lambda\left(k_{2} \int_{t-\tau(t)}^{t} x^{T}(s) x(s) d s\right)^{(1+\eta) / 2}+s_{4} x^{T}(t) \\
& \cdot E E^{T} x(t)+s_{4}^{-1} \omega^{T}(t) \omega(t)+2 x^{T}(t) \\
& \cdot\left[-\frac{s_{4}}{2} E E^{T} x(t)-\frac{s_{4}^{-1} d}{2\|x(t)\|^{2}} x(t)\right] \leq x^{T}(t)\left[A^{T}\right. \\
& \left.+A-2 k_{1} I+k_{2} I+\left(s_{1} \alpha+s_{1}^{-1}\right) I+s_{2} B B^{T}+s_{3}^{-1} I\right] \\
& \cdot x(t)+x^{T}(t-\tau(t))\left[\left(s_{2}^{-1} L+s_{3} \beta L\right) I\right. \\
& \left.-k_{2}(1-\mu) I\right] x(t-\tau(t))-2 \lambda\left|x(t)^{T} x(t)\right|^{(1+\eta) / 2} \\
& -2 \lambda\left(k_{2} \int_{t-\tau(t)}^{t} x^{T}(s) x(s) d s\right)^{(1+\eta) / 2}+s_{4} x^{T}(t) \\
& \cdot E E^{T} x(t)+s_{4}^{-1} d+2 x^{T}(t)\left[-\frac{s_{4}}{2} E E^{T} x(t)\right. \\
& \left.-\frac{s_{4}^{-1} d}{2\|x(t)\|^{2}} x(t)\right] \leq-2 \lambda\left|x^{T}(t) x(t)\right|^{(1+\eta) / 2} \\
& -2 \lambda\left(k_{2} \int_{t-\tau(t)}^{t} x^{T}(s) x(s) d s\right)^{(1+\eta) / 2}
\end{aligned}
$$




$$
\begin{aligned}
& \leq-2 \lambda\left[x^{T}(t) x(t)\right. \\
& \left.+k_{2} \int_{t-\tau(t)}^{t} x^{T}(s) x(s) d s\right]^{(1+\eta) / 2}=-2 \lambda V^{(1+\eta) / 2}(t) .
\end{aligned}
$$

It then follows from Lemma 3 that

$$
\begin{aligned}
V^{1-(1+\eta) / 2}(t) \leq & V^{1-(1+\eta) / 2}\left(t_{0}\right) \\
& -2 \lambda\left(1-\frac{1+\eta}{2}\right)\left(t-t_{0}\right),
\end{aligned}
$$

$$
t_{0} \leq t \leq T
$$

and $V(t) \equiv 0, \forall t \geq T$, with $T$ given by

$$
T=t_{0}+\frac{V^{1-(1+\eta) / 2}\left(t_{0}\right)}{2 \lambda(1-(1+\eta) / 2)}=t_{0}+\frac{V^{(1-\eta) / 2}\left(t_{0}\right)}{\lambda(1-\eta)}
$$

Therefore, we obtain that

$$
\|x(t)\| \equiv 0, \quad t \geq T
$$

Then system (1) is finite-time stable with control input $u(t)$ in (5), and the finite time is estimated by

$$
T=t_{0}+\frac{V^{(1-\eta) / 2}\left(t_{0}\right)}{\lambda(1-\eta)}
$$

The proof is completed.

Remark 7. In the paper, the finite-time stability problem of nonlinear systems with time-varying delays, parameter uncertainties, and external disturbances is addressed. Although the finite-time stability problem of some kinds of systems with time delays is studied in $[30,32]$, the parameter uncertainties and external disturbances are not taken into account in these papers. So our methods are more general compared with the existing results.

Particularly, system (1) is linear if we let $f(x)=x$. For such a linear system, we can obtain the following corollary by choosing $L=1$ in condition (ii) of Theorem 6.

Corollary 8. Assume that there exist constants $s_{1}, s_{2}, s_{3}>0$ and $k_{1}, k_{2}>0$ such that

(i) $A^{T}+A-2 k_{1} I+k_{2} I+s_{2} B B^{T}+s_{3}^{-1} I+\left(s_{1} \alpha+s_{1}^{-1}\right) I \leq 0$;

(ii) $s_{2}^{-1}+s_{3} \beta-k_{2}(1-\mu) \leq 0$;

then the linear system is finite-time stable with control input $u(t)$ in (5), and the finite time is estimated by

$$
T=t_{0}+\frac{V^{(1-\eta) / 2}\left(t_{0}\right)}{\lambda(1-\eta)} .
$$

Based on Theorem 6, one can easily get the following corollary.
Corollary 9. Assume that there exist constants $s_{1}, s_{2}, s_{3}>0$ such that

$$
\geq \frac{\lambda_{\text {max }}\left(A+A^{T}\right)+s_{2} \lambda_{\text {max }}\left(B B^{T}\right)+s_{3}^{-1}+s_{1} \alpha+s_{1}^{-1}+k_{2}}{2},
$$

$$
k_{2} \geq \frac{\left(s_{2}^{-1}+s_{3} \beta\right) L}{1-\mu}
$$

then system (1) is finite-time stable with control input $u(t)$ in (5), and the finite time is estimated by

$$
T=t_{0}+\frac{V^{(1-\eta) / 2}\left(t_{0}\right)}{\lambda(1-\eta)}
$$

\section{Simulation Examples}

In this section, two examples are provided to verify the effectiveness of the results obtained in the previous section.

Example 1. Consider a two-dimensional uncertain nonlinear system without external disturbances:

$$
\dot{x}(t)=(A+\Delta A) x(t)+(B+\Delta B) f(x(t-\tau(t))),
$$

where $x(t)=\left(x_{1}(t), x_{2}(t)\right)^{T}, \tau(t)=0.4+0.1 \cos t$,

$$
\begin{aligned}
A & =\left(\begin{array}{ll}
1 & 0 \\
0 & 1
\end{array}\right), \\
\Delta A & =\left(\begin{array}{cc}
0.2 & 0 \\
0 & 0.2
\end{array}\right), \\
B & =\left(\begin{array}{cc}
-2.5 & 0.2 \\
0.1 & -1.5
\end{array}\right), \\
\Delta B & =\left(\begin{array}{ll}
0.5 & 0.5 \\
0.5 & 0.5
\end{array}\right),
\end{aligned}
$$

and $f(x(t))=\tanh (x(t))$.

Choose $\alpha=0.04, \beta=1$, and $\mu=0.5$. It is obvious that $\Delta A^{T} \Delta A \leq \alpha I, \Delta B^{T} \Delta B \leq \beta I$, and $\dot{\tau}(t) \leq \mu<1$. We can select $L=1$. Under the initial condition $x_{1}(s)=0.5$ and $x_{2}(s)=$ $-0.5, \forall s \in[-1,0)$, the state trajectories of $x_{1}(t)$ and $x_{2}(t)$ of system (26) are shown in Figure 1.

Now, we consider system (26) with control input; the resulting system is given by

$$
\begin{aligned}
\dot{x}(t)= & (A+\Delta A) x(t)+(B+\Delta B) f(x(t-\tau(t))) \\
& +u(t) .
\end{aligned}
$$

For numerical simulation, we can select $\lambda=0.1, \eta=0.8$, $s_{1}=1, s_{2}=1$, and $s_{3}=1$. Based on Corollary 9, we have 


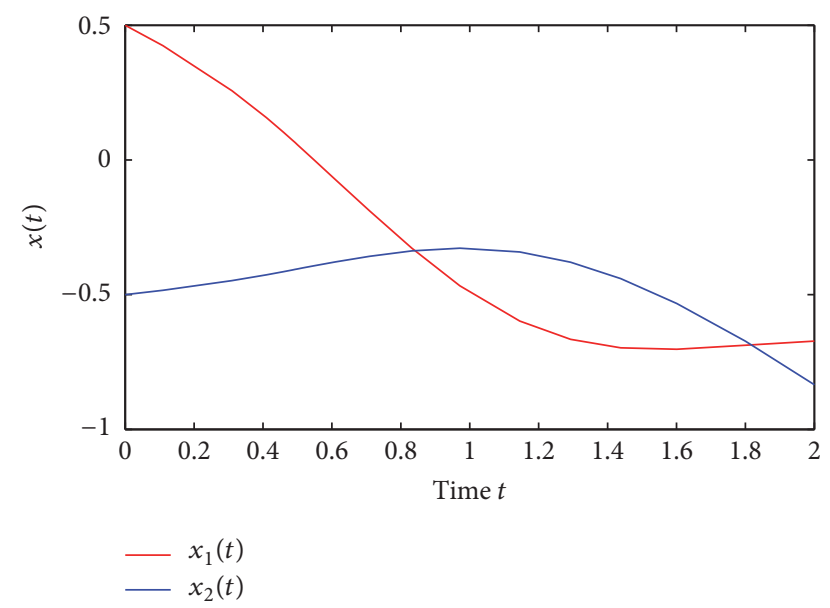

FIGURE 1: State trajectories of system (26) without control input under the initial conditions $x_{1}(s)=0.5$ and $x_{2}(s)=-0.5, \forall s \in$ $[-1,0)$.

$k_{2} \geq 4$ and $k_{1} \geq 7.6819$. We choose $k_{2}=4$ and $k_{1}=8$. So the controller $u(t)=u_{1}(t)=\left(u_{11}(t), u_{12}(t)\right)^{T}$ is designed as

$$
\begin{aligned}
& u_{11}(t)=-8 x_{1}(t)-0.1 \operatorname{sign}\left(x_{1}(t)\right)\left|x_{1}(t)\right|^{0.8}-0.1 \\
& \cdot 4^{0.9}\left(\int_{t-(0.4+0.1 \cos t)}^{t}\left(x_{1}^{2}(s)+x_{2}^{2}(s)\right) d s\right)^{0.9} \\
& \cdot \frac{x_{1}(t)}{x_{1}^{2}(t)+x_{2}^{2}(t)}, \\
& u_{12}(t)-8 x_{2}(t)-0.1 \operatorname{sign}\left(x_{2}(t)\right)\left|x_{2}(t)\right|^{0.8}-0.1 \\
& \cdot 4^{0.9}\left(\int_{t-(0.4+0.1 \cos t)}^{t}\left(x_{1}^{2}(s)+x_{2}^{2}(s)\right) d s\right)^{0.9} \\
& \cdot \frac{x_{2}(t)}{x_{1}^{2}(t)+x_{2}^{2}(t)} .
\end{aligned}
$$

Under the initial condition $x_{1}(s)=0.5$ and $x_{2}(s)=-0.5$, $\forall s \in[-1,0)$, the state trajectories of $x_{1}(t)$ and $x_{2}(t)$ of system (28) are shown in Figure 2. Hence, system (26) is finite-time stable with control input $u(t)$ defined in (29).

Example 2. Consider a three-dimensional uncertain nonlinear system with external disturbances:

$$
\begin{aligned}
\dot{x}(t)= & (A+\Delta A) x(t)+(B+\Delta B) f(x(t-\tau(t))) \\
& +E \omega(t),
\end{aligned}
$$

where $x(t)=\left(x_{1}(t), x_{2}(t), x_{3}(t)\right)^{T}, \tau(t)=0.2, E=(0,0,1)^{T}$, $\omega(t)=\sin t$,

$$
A=\left(\begin{array}{lll}
1 & 0 & 0 \\
0 & 1 & 0 \\
0 & 0 & 1
\end{array}\right),
$$

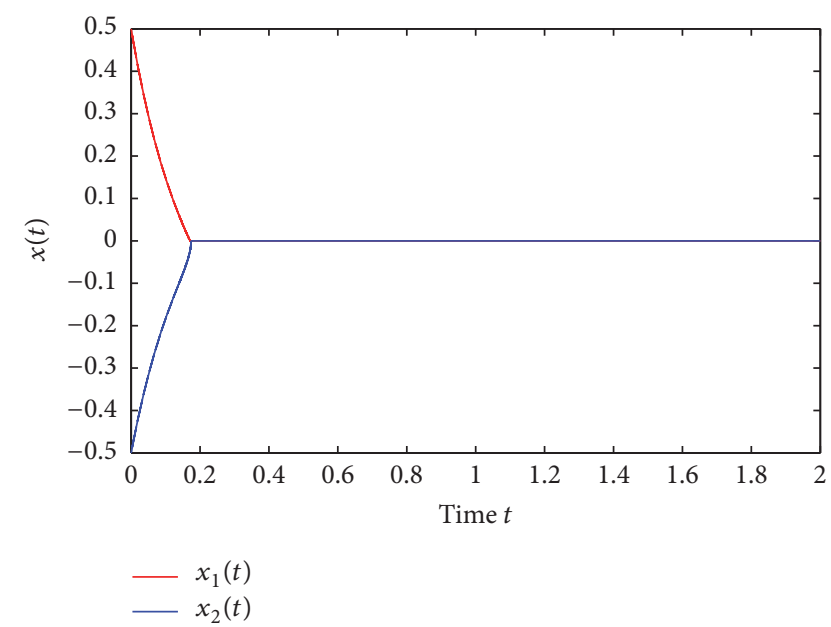

Figure 2: State trajectories of system (26) with control input (29) under the initial conditions $x_{1}(s)=0.5$ and $x_{2}(s)=-0.5, \forall s \in$ $[-1,0)$.

$$
\begin{aligned}
\Delta A & =\left(\begin{array}{ccc}
0.2 & 0 & 0 \\
0 & 0.2 & 0 \\
0 & 0 & 0.2
\end{array}\right), \\
B & =\left(\begin{array}{ccc}
1 & 0.5 & -0.8 \\
-0.6 & 1.5 & 0.5 \\
0.4 & 0.6 & 1 \\
0 & &
\end{array}\right), \\
\Delta B & =\left(\begin{array}{lll}
0.2 & 0.2 & 0.2 \\
0.2 & 0.2 & 0.2 \\
0.2 & 0.2 & 0.2
\end{array}\right),
\end{aligned}
$$

and $f(x(t))=\tanh (x(t))$.

Choose $\alpha=0.04, \beta=0.5, \mu=0.5$, and $d=1$. It is obvious that $\Delta A^{T} \Delta A \leq \alpha I, \Delta B^{T} \Delta B \leq \beta I, \omega^{T}(t) \omega(t) \leq d$, and $\dot{\tau}(t) \leq \mu<1$. We can select $L=1$. Under the initial condition $x_{1}(s)=-0.6, x_{2}(s)=0.5$, and $x_{3}(s)=-1.5, \forall s \in[-1,0)$, the state trajectories of variables $x_{1}(t), x_{2}(t)$, and $x_{3}(t)$ of system (30) are shown in Figure 3.

System (30) with control input is given as

$$
\begin{aligned}
\dot{x}(t)= & (A+\Delta A) x(t)+(B+\Delta B) f(x(t-\tau(t))) \\
& +E \omega(t)+u(t) .
\end{aligned}
$$

For numerical simulation, we can select $\lambda=0.5, \eta=0.6$, $s_{1}=1, s_{2}=1, s_{3}=1$, and $s_{4}=1$. Based on Corollary 8 , we 


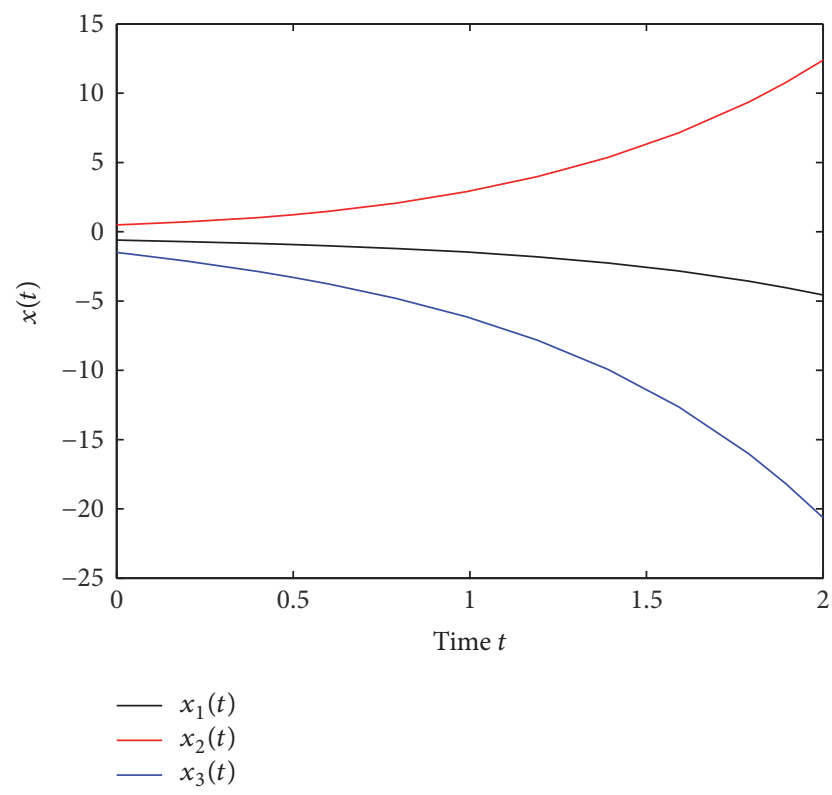

FIGURE 3: State trajectories of system (30) without control input under the initial conditions $x_{1}(s)=-0.6, x_{2}(s)=0.5$, and $x_{3}(s)=$ $-1.5, \forall s \in[-1,0)$.

have $k_{2} \geq 3$ and $k_{1} \geq 5.3059$. We choose $k_{2}=3$ and $k_{1}=6$. So the controller $u(t)=u_{1}(t)+u_{2}(t)$ is designed as

$$
\begin{aligned}
& u_{1}(t)=\left(u_{11}(t), u_{12}(t), u_{13}(t)\right)^{T}, \\
& u_{2}(t)=\left(u_{21}(t), u_{22}(t), u_{23}(t)\right)^{T},
\end{aligned}
$$

where

$$
\begin{aligned}
& u_{11}(t)=-6 x_{1}(t)-0.5 \operatorname{sign}\left(x_{1}(t)\right)\left|x_{1}(t)\right|^{0.6}-0.5 \\
& \cdot 3^{0.8}\left(\int_{t-0.2}^{t}\left(x_{1}^{2}(s)+x_{2}^{2}(s)+x_{3}^{2}(s)\right) d s\right)^{0.8} \\
& \cdot \frac{x_{1}(t)}{x_{1}^{2}(t)+x_{2}^{2}(t)+x_{3}^{2}(t)}, \\
& u_{12}(t)=-6 x_{2}(t)-0.5 \operatorname{sign}\left(x_{2}(t)\right)\left|x_{2}(t)\right|^{0.6}-0.5 \\
& \cdot 3^{0.8}\left(\int_{t-0.2}^{t}\left(x_{1}^{2}(s)+x_{2}^{2}(s)+x_{3}^{2}(s)\right) d s\right)^{0.8} \\
& \cdot \frac{x_{2}(t)}{x_{1}^{2}(t)+x_{2}^{2}(t)+x_{3}^{2}(t)}, \\
& u_{13}(t)=-6 x_{3}(t)-0.5 \operatorname{sign}\left(x_{3}(t)\right)\left|x_{3}(t)\right|^{0.6}-0.5 \\
& \cdot 3^{0.8}\left(\int_{t-0.2}^{t}\left(x_{1}^{2}(s)+x_{2}^{2}(s)+x_{3}^{2}(s)\right) d s\right)^{0.8} \\
& \cdot \frac{x_{2}(t)}{x_{1}^{2}(t)+x_{2}^{2}(t)+x_{3}^{2}(t)},
\end{aligned}
$$

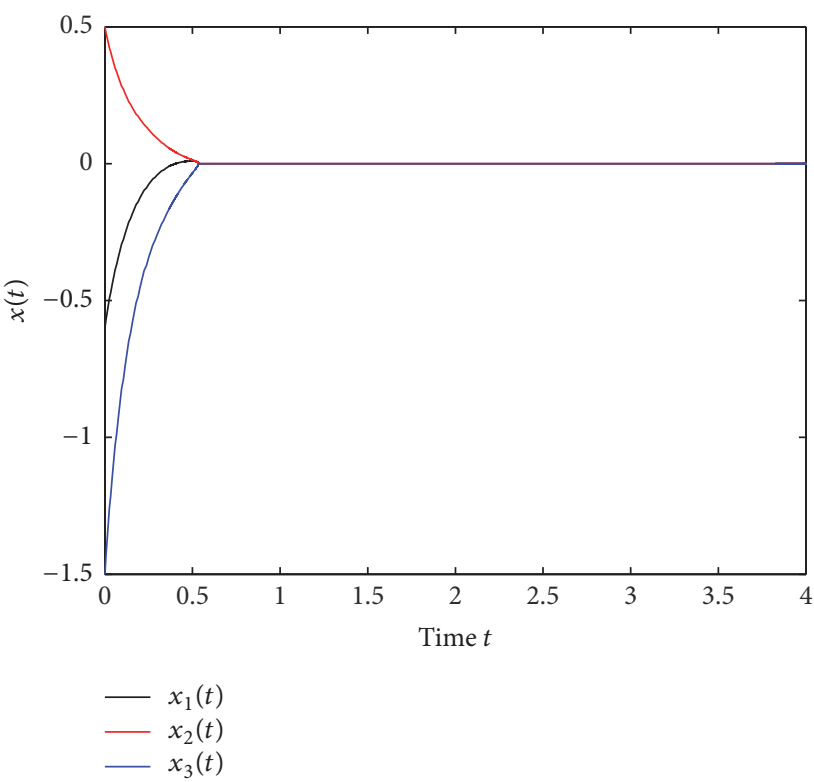

FIGURE 4: State trajectories of system (30) with control input (34) under the initial conditions $x_{1}(s)=-0.6, x_{2}(s)=0.5$, and $x_{3}(s)=$ $-1.5, \forall s \in[-1,0)$.

$$
\begin{aligned}
& u_{21}(t)=-\frac{x_{1}(t)}{2\left(x_{1}^{2}(t)+x_{2}^{2}(t)+x_{3}^{2}(t)\right)} \\
& u_{22}(t)=-\frac{x_{2}(t)}{2\left(x_{1}^{2}(t)+x_{2}^{2}(t)+x_{3}^{2}(t)\right)}, \\
& u_{23}(t)=-\frac{x_{3}(t)}{2}-\frac{x_{3}(t)}{2\left(x_{1}^{2}(t)+x_{2}^{2}(t)+x_{3}^{2}(t)\right)} .
\end{aligned}
$$

Under the initial condition $x_{1}(s)=-0.6, x_{2}(s)=0.5$, and $x_{3}(s)=-1.5, \forall s \in[-1,0)$, the state trajectories of $x_{1}(t), x_{2}(t)$, and $x_{3}(t)$ of system (32) are shown in Figure 4. Hence, system (30) is finite-time stable with control input $u(t)$ given in (34).

\section{Conclusion}

In this paper, we have investigated the finite-time stability problem for a class of uncertain nonlinear systems with time-varying delay and external disturbances. By using the Lyapunov stability theory, sufficient conditions guaranteeing the finite-time stability of the considered systems have been developed. Two simulation examples have been provided to validate the effectiveness and correctness of the proposed theoretical results. Future work will focus on the finite-time stability for uncertain nonlinear systems with distributed delay.

\section{Conflicts of Interest}

The authors declare that they have no conflicts of interest. 


\section{Acknowledgments}

This work was supported by the National Natural Science Foundation of China (11301308 and 61673247), the Research Fund for Distinguished Young Scholars and Excellent Young Scholars of Shandong Province (ZR201702100145 and JQ201719), and Shandong Province Key Laboratory of Medical Physics and Image Processing Technology.

\section{References}

[1] W. M. Haddad and V. Chellaboina, "Stability theory for nonnegative and compartmental dynamical systems with time delay," Systems \& Control Letters, vol. 51, no. 5, pp. 355-361, 2004.

[2] H. Zhang and Z. Liu, "Stability analysis for linear delayed systems via an optimally dividing delay interval approach," Automatica, vol. 47, no. 9, pp. 2126-2129, 2011.

[3] X. Li and J. Wu, "Stability of nonlinear differential systems with state-dependent delayed impulses," Automatica, vol. 64, pp. 6369, 2016.

[4] J. Tian and S. Zhong, "Improved delay-dependent stability criteria for neural networks with two additive time-varying delay components," Neurocomputing, vol. 77, no. 1, pp. 114-119, 2012.

[5] X. Li, R. Rakkiyappan, and N. Sakthivel, "Non-fragile synchronization control for Markovian jumping complex dynamical networks with probabilistic time-varying coupling delays," Asian Journal of Control, vol. 17, no. 5, pp. 1678-1695, 2015.

[6] W. Wang and S. Zhong, "Delay-dependent stability criteria for genetic regulatory networks with time-varying delays and nonlinear disturbance," Communications in Nonlinear Science and Numerical Simulation, vol. 17, no. 9, pp. 3597-3611, 2012.

[7] X. Li and S. Song, "Stabilization of delay systems: delaydependent impulsive control," Institute of Electrical and Electronics Engineers Transactions on Automatic Control, vol. 62, no. 1, pp. 406-411, 2017.

[8] H. Zhang and G. Wang, "New criteria of global exponential stability for a class of generalized neural networks with timevarying delays," Neurocomputing, vol. 70, no. 13-15, pp. 24862494, 2007.

[9] X. Li and X. Fu, "Effect of leakage time-varying delay on stability of nonlinear differential systems," Journal of The Franklin Institute, vol. 350, no. 6, pp. 1335-1344, 2013.

[10] B. Øksendal, Stochastic Differential Equations: An Introduction with Applications, Springer, Berlin, Germany, 6th edition, 1998.

[11] R. Saravanakumar, M. Syed Ali, and H. R. Karimi, "Robust Ho control of uncertain stochastic Markovian jump systems with mixed time-varying delays," International Journal of Systems Science, vol. 48, no. 4, pp. 862-872, 2017.

[12] A. Weinmann, Uncertain Models and Robust Control, Springer, Vienna, Austria, 1991.

[13] M. Syed Ali, N. Gunasekaran, and M. Esther Rani, "Robust stability of hopfield delayed neural networks via an augmented L-K functional," Neurocomputing, vol. 234, pp. 198-204, 2017.

[14] X. Wang, C. D. Li, and T. W. Huang, "Delay-dependent robust stability and stabilization of uncertain memristive delay neural networks," Neurocomputing, vol. 140, pp. 155-161, 2014.

[15] M. S. Ali and P. Balasubramaniam, "Exponential stability of time-delay systems with nonlinear uncertainties," International Journal of Computer Mathematics, vol. 87, no. 6, pp. 1363-1373, 2010.
[16] X. Li and J. Cao, "An impulsive delay inequality involving unbounded time-varying delay and applications," Institute of Electrical and Electronics Engineers Transactions on Automatic Control, vol. 62, no. 7, pp. 3618-3625, 2017.

[17] I. Stamova, T. Stamov, and X. Li, "Global exponential stability of a class of impulsive cellular neural networks with supremums," International Journal of Adaptive Control and Signal Processing, vol. 28, no. 11, pp. 1227-1239, 2014.

[18] X. Li, M. Bohner, and C.-K. Wang, "Impulsive differential equations: periodic solutions and applications," Automatica, vol. 52, pp. 173-178, 2015.

[19] S. P. Bhat and D. S. Bernstein, "Finite-time stability of continuous autonomous systems," SIAM Journal on Control and Optimization, vol. 38, no. 3, pp. 751-766, 2000.

[20] Y. Hong, J. Huang, and Y. Xu, "On an output feedback finitetime stabilization problem," Institute of Electrical and Electronics Engineers Transactions on Automatic Control, vol. 46, no. 2, pp. 305-309, 2001.

[21] A. Polyakov, "Nonlinear feedback design for fixed-time stabilization of linear control systems," Institute of Electrical and Electronics Engineers Transactions on Automatic Control, vol. 57, no. 8, pp. 2106-2110, 2012.

[22] S. P. Bhat and D. S. Bernstein, "Geometric homogeneity with applications to finite-time stability," Mathematics of Control, Signals, and Systems, vol. 17, no. 2, pp. 101-127, 2005.

[23] S. G. Nersesov, W. M. Haddad, and Q. Hui, "Finite-time stabilization of nonlinear dynamical systems via control vector Lyapunov functions," Journal of The Franklin Institute, vol. 345, no. 7, pp. 819-837, 2008.

[24] Y. Hong and Z.-P. Jiang, "Finite-time stabilization of nonlinear systems with parametric and dynamic uncertainties," Institute of Electrical and Electronics Engineers Transactions on Automatic Control, vol. 51, no. 12, pp. 1950-1956, 2006.

[25] E. Moulay and W. Perruquetti, "Finite time stability and stabilization of a class of continuous systems," Journal of Mathematical Analysis and Applications, vol. 323, no. 2, pp. 1430-1443, 2006.

[26] X. Liu, N. Jiang, J. Cao, S. Wang, and Z. Wang, "Finitetime stochastic stabilization for BAM neural networks with uncertainties," Journal of The Franklin Institute, vol. 350, no. 8, pp. 2109-2123, 2013.

[27] P. M. Aghababa and H. P. Aghababa, "Adaptive finite-time stabilization of uncertain non-autonomous chaotic electromechanical gyrostat systems with unknown parameters," Mechanics Research Communications, vol. 38, no. 7, pp. 500-505, 2011.

[28] X. Huang, W. Lin, and B. Yang, "Global finite-time stabilization of a class of uncertain nonlinear systems," Automatica, vol. 41, no. 5, pp. 881-888, 2005.

[29] I. Karafyllis, "Finite-time global stabilizaton by means of timevarying distributed delay feedback," SIAM Journal on Control and Optimization, vol. 45, no. 1, pp. 320-342, 2006.

[30] E. Moulay, M. Dambrine, N. Yeganefar, and W. Perruquetti, "Finite-time stability and stabilization of time-delay systems," Systems \& Control Letters, vol. 57, no. 7, pp. 561-566, 2008.

[31] D. Efimov, A. Polyakov, E. Fridman, W. Perruquetti, and J.P. Richard, "Comments on finite-time stability of time-delay systems," Automatica, vol. 50, no. 7, pp. 1944-1947, 2014.

[32] R. Yang and Y. Wang, "Finite-time stability analysis and Ho control for a class of nonlinear time-delay Hamiltonian systems," Automatica, vol. 49, no. 2, pp. 390-401, 2013. 
[33] B. Liu, D. J. Hill, and Z. J. Sun, "Mixed Kscript-Dissipativity and Stabilization to ISS for Impulsive Hybrid Systems," IEEE Transactions on Circuits and Systems II: Express Briefs, vol. 62, no. 8, pp. 791-795, 2015.

[34] W. M. Haddad, S. G. Nersesov, and L. Du, "Finite-time stability for time-varying nonlinear dynamical systems," in Proceedings of the 2008 American Control Conference, ACC, pp. 4135-4139, June 2008.

[35] X. Yang and J. Cao, "Finite-time stochastic synchronization of complex networks," Applied Mathematical Modelling: Simulation and Computation for Engineering and Environmental Systems, vol. 34, no. 11, pp. 3631-3641, 2010.

[36] Y. Liu, C. Li, T. Huang, and X. Wang, "Robust adaptive lag synchronization of uncertain fuzzy memristive neural networks with time-varying delays," Neurocomputing, vol. 190, pp. 188196, 2016

[37] Y. Wu, J. Cao, Q. Li, A. Alsaedi, and F. E. Alsaadi, "Finite-time synchronization of uncertain coupled switched neural networks under asynchronous switching," Neural Networks, vol. 85, pp. 128-139, 2017.

[38] J. Huang, C. Li, T. Huang, and X. He, "Finite-time lag synchronization of delayed neural networks," Neurocomputing, vol. 139, pp. 145-149, 2014.

[39] Y. Li, X. Yang, and L. Shi, "Finite-time synchronization for competitive neural networks with mixed delays and nonidentical perturbations," Neurocomputing, vol. 185, pp. 242-253, 2016.

[40] X. Lv and X. Li, "Finite time stability and controller design for nonlinear impulsive sampled-data systems with applications," ISA Transactions, vol. 70, pp. 30-36, 2017.

[41] M. S. Ali and J. Yogambigai, "Finite-time robust stochastic synchronization of uncertain Markovian complex dynamical networks with mixed time-varying delays and reactiondiffusion terms via impulsive control," Journal of The Franklin Institute, vol. 354, no. 5, pp. 2415-2436, 2017.

[42] M. S. Ali, J. Yogambigai, and J. Cao, "Synchronization of masterslave Markovian switching complex dynamical networks with time-varying delays in nonlinear function via sliding mode control," Acta Mathematica Scientia B, vol. 37, no. 2, pp. 368384, 2017.

[43] L. Wang, Y. Shen, and Y. Sheng, "Finite-time robust stabilization of uncertain delayed neural networks with discontinuous activations via delayed feedback control," Neural Networks, vol. 76, pp. 46-54, 2016.

[44] S. Boyd, L. El Ghaoui, E. Feron, and V. Balakrishnan, Linear matrix inequalities in system and control theory, vol. 15 of SIAM Studies in Applied Mathematics, SIAM, Philadelphia, Pa, USA, 1994.

[45] C. Qian and J. Li, "Robust exponential stability of uncertain impulsive delays differential systems," IEEE Transactions on Automatic Control, vol. 50, pp. 885-890, 2005. 


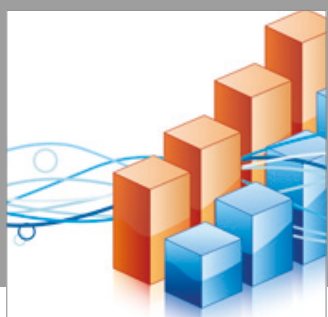

Advances in

Operations Research

vatersals

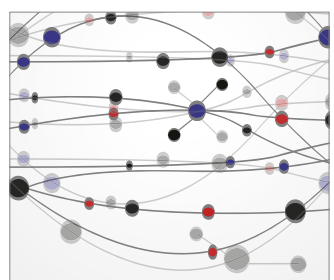

\section{The Scientific} World Journal
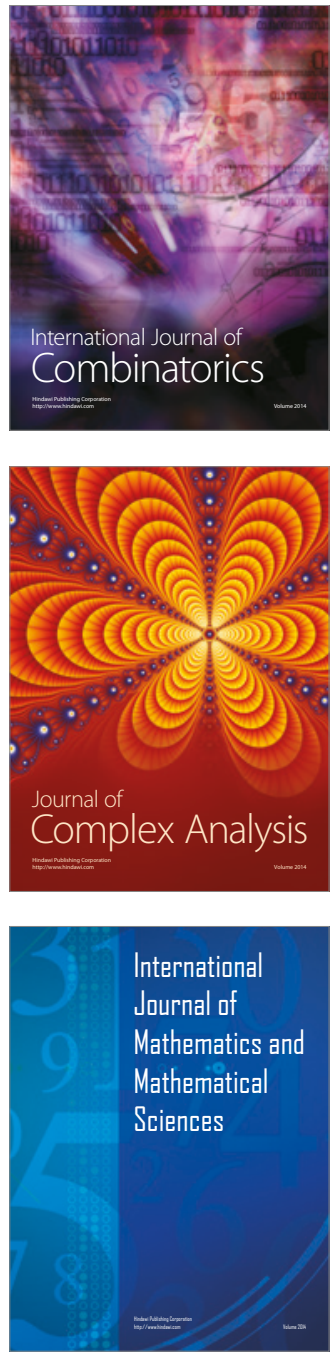
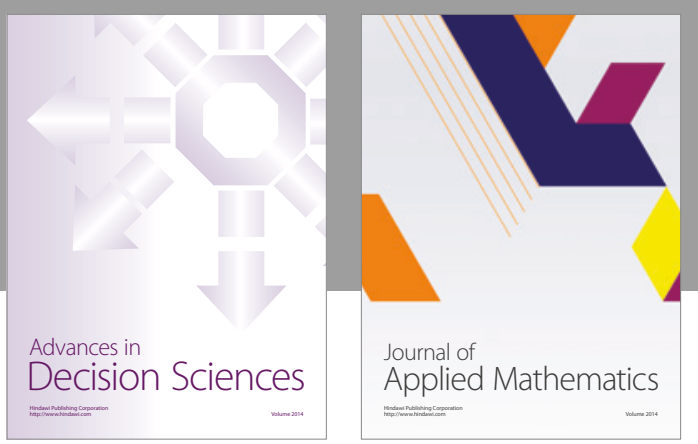

Algebra

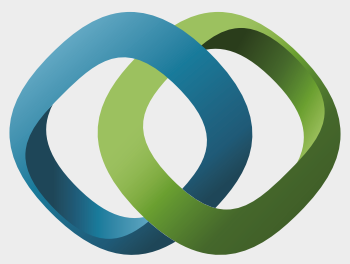

\section{Hindawi}

Submit your manuscripts at

https://www.hindawi.com
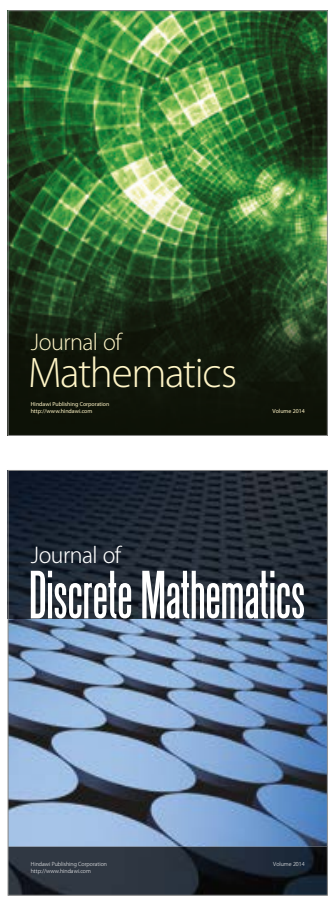

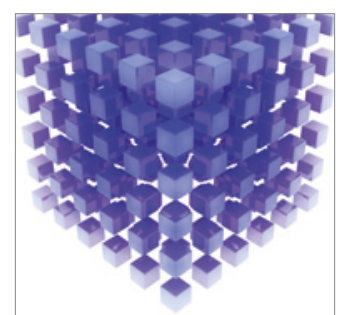

Mathematical Problems in Engineering
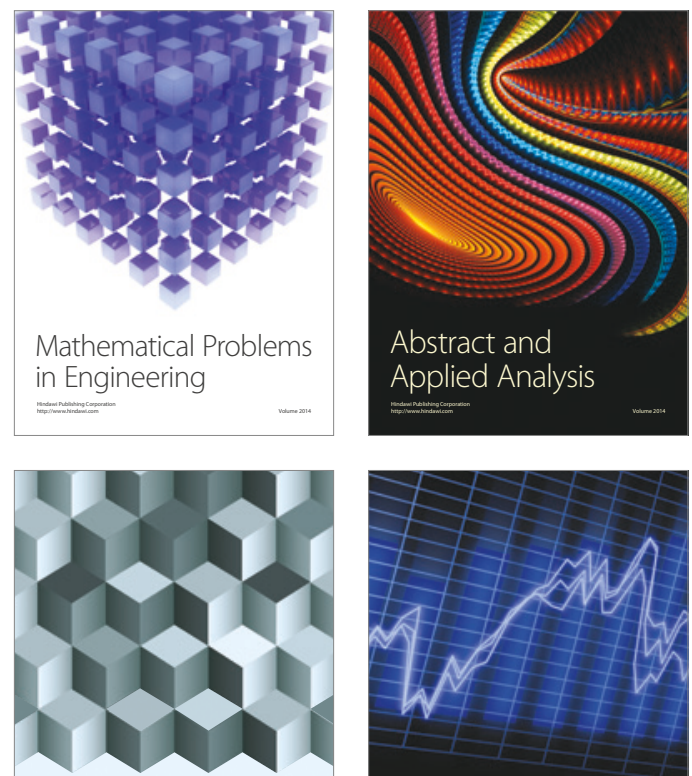

Journal of

Function Spaces

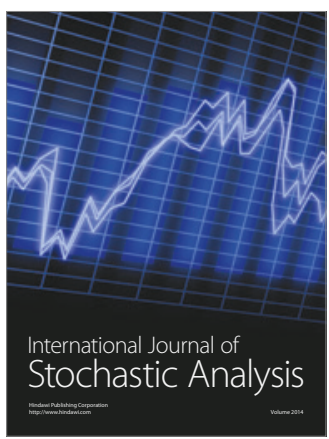

Probability and Statistics
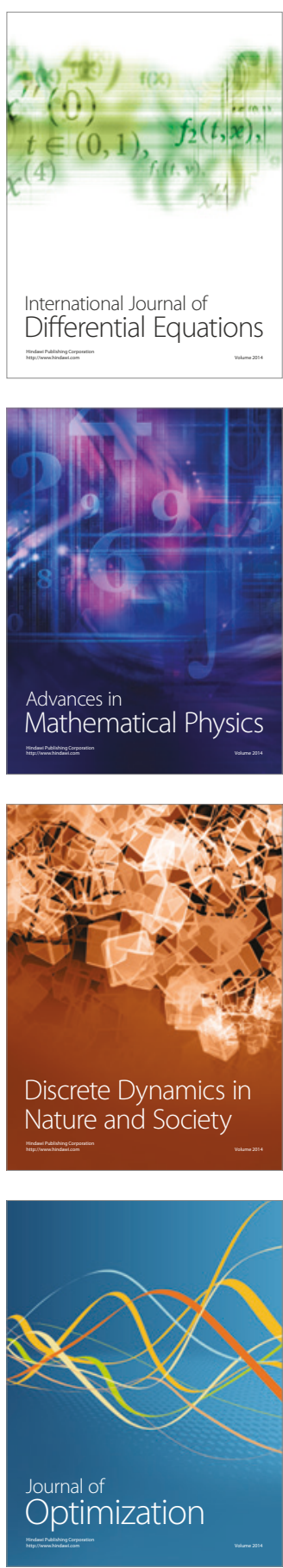